\title{
Matériaux sélectifs pour la conversion photothermique de l'énergie solaire
}

\author{
J. Spitz et A. Aubert \\ Laboratoire d'Etudes des Matériaux Minces \\ Centre d'Etudes Nucléaires de Grenoble \\ 85 X, 38041 Grenoble Cedex, France
}

\author{
J. M. Behaghel, S. Berthier, J. Lafait et J. Rivory \\ Laboratoire d'Optique des Solides, Université Pierre et Marie-Curie
} 4, place Jussieu, 75230 Paris Cedex 05, France

(Reçu le 6 juillet 1978, révisé le 23 octobre 1978, accepté le 25 octobre 1978)

\begin{abstract}
Résumé. - On montre l'intérêt des surfaces sélectives pour la conversion photothermique de l'énergie solaire. Les divers phénomènes physiques susceptibles de conduire à la sélectivité spectrale sont décrits. On définit différents critères qui doivent intervenir lors du choix d'une surface sélective. On rend enfin compte d'un certain nombre de résultats obtenus avec des matériaux tels que le noir de chrome, les carbures et les nitrures de titane et l'oxyde d'indium dopé.
\end{abstract}

\begin{abstract}
The interest in the selective surfaces for the photothermal conversion of solar energy is demonstrated. The various physical phenomena which lead to the spectral selectivity are briefly investigated. The different criteria which may be considered at the time of chosing a selective surface are described. Finaly some of the results obtained with materials like black chrome, the carbides or nitrides of titanium and the doped indium oxide are described and discussed.
\end{abstract}

1. Introduction. - La conversion photothermique de l'énergie solaire peut constituer un apport d'énergie non négligeable pour un grand nombre d'applications thermiques, thermomécaniques et thermochimiques à condition que des rendements de conversion élevés puissent être atteints dans une gamme de températures relativement étendue.

Quatre catégories d'applications se dégagent en fonction de la température :

- applications aux basses températures solaires $\left(T<60^{\circ} \mathrm{C}\right)$ : chauffage de l'eau sanitaire, chauffage des piscines...,

- applications aux moyennes températures solaires $\left(60^{\circ} \mathrm{C}<T<150^{\circ} \mathrm{C}\right)$ : chauffage des habitations, réfrigération, climatisation, dessalement de l'eau de mer, production d'énergie mécanique...,

- applications aux hautes températures solaires $\left(150^{\circ} \mathrm{C}<T<800^{\circ} \mathrm{C}\right)$ : production d'énergie mécanique, production de vapeur, dissociation catalytique de l'eau...,

- applications aux très hautes températures solaires $\left(T>800^{\circ} \mathrm{C}\right)$ : dissociation thermique de l'eau, magnétohydrodynamique, thermo-électricité).

Pour chacune de ces applications c'est bien souvent les performances, ou mieux, le rapport performance/ coût du capteur qui décide de l'intérêt du système de conversion de l'énergie solaire par rapport aux autres sources d'énergie éventuellement disponibles. Le prix du capteur constitue, en effet, la majeure partie du coût total de l'installation solaire. Les performances du capteur dans un site donné dépendent essentiellement de sa conception et des propriétés optiques et thermiques des matériaux qui le composent. Sans négliger l'importance des systèmes concentrateurs, des fluides de transfert et des milieux de stockage de la chaleur ainsi que des problèmes posés par la convection et la conduction, nous nous limiterons dans cet article à considérer les problèmes radiatifs se posant au niveau du capteur. Nous montrerons comment on peut accroître ses performances en optimisant les propriétés optiques des matériaux constituant la surface de son absorbeur ou sa couverture, afin d'obtenir un comportement spectral sélectif de ces surfaces. Nous présenterons enfin quelques solutions intéressantes à ce problème des matériaux sélectifs obtenus dans nos laboratoires : dépôts de chrome noir, carbures et nitrures de titane sans et avec structures en surface, oxyde d'indium dopé.

2. Définition des surfaces sélectives. - Les principaux effets intervenant dans un capteur sont réunis 
sur la figure 1. Seule une fraction de l'énergie incidente est transmise au fluide de transfert circulant dans l'absorbeur. Une part de l'énergie incidente est perdue par réflexion sur la couverture du capteur (s'il en possède une) et sur la surface de l'absorbeur. Ce sont les pertes optiques. Une autre part de l'énergie thermique produite par l'absorption des photons incidents est perdue par transfert vers l'environnement du capteur par convection, conduction et rayonnement. Ce sont les pertes thermiques. Les pertes thermiques par convection et conduction sont en première approximation proportionnelles à la température. Les pertes thermiques par rayonnement sont par contre proportionnelles à la puissance quatrième de la température, conformément à la loi de StefanBoltzmann.

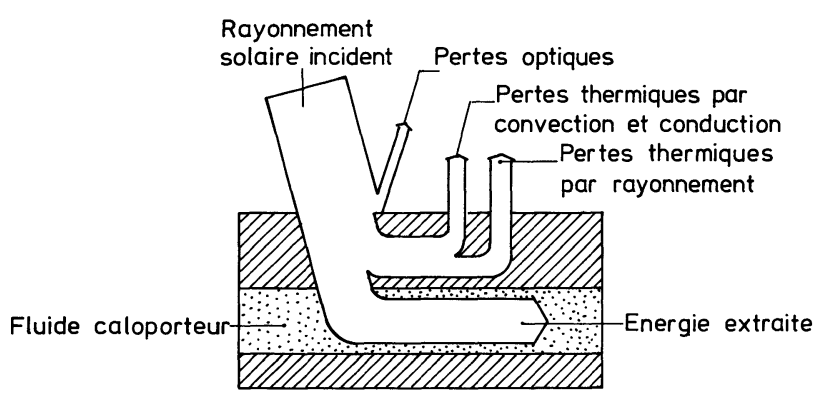

Fig. 1. - Bilan énergétique d'un capteur.

[Energy balance of a collector.]

L'équation suivante exprime le bilan énergétique simplifié d'un capteur à une température et dans des conditions météorologiques données :

$$
\begin{aligned}
Q_{\mathrm{u}}= & S_{\mathrm{c}} \cdot a \cdot E^{\mathrm{s}}-S_{\mathrm{a}} \cdot e \cdot \sigma\left(T_{\mathrm{a}}^{4}-T_{0}^{4}\right)- \\
& -S_{\mathrm{a}} \cdot H \cdot\left(T_{\mathrm{a}}-T_{0}\right)
\end{aligned}
$$

où

$Q_{\mathrm{u}}=$ puissance extraite $\ldots \ldots \ldots \ldots \mathrm{W}$

$S_{\mathrm{c}}=$ surface de collection du flux solaire incident ............ $\mathrm{m}^{2}$

$a=$ absorptivité du capteur (compte tenu des diverses pertes par réflexion et transmission avant l'absorbeur)

$E^{\mathrm{s}}=$ éclairement solaire au sol.... W. W. $\mathrm{m}^{-2}$

$S_{\mathrm{a}}=$ surface de l'absorbeur...... $\mathrm{m}^{2}$

$e=$ émissivité de la totalité du capteur

$T_{\mathrm{a}}=$ température de l'absorbeur $\ldots \mathrm{K}$

$T_{0}=$ température équivalente de l'environnement............ $\mathrm{K}$

$H=$ facteur traduisant les effets de conduction et de convection

$\sigma=$ constante de Stefan-Boltzmann $\mathrm{W} \cdot \mathrm{m}^{-2} \cdot \mathrm{K}^{-4}$

La figure 2 représente les variations des différentes pertes du capteur en fonction de la température.

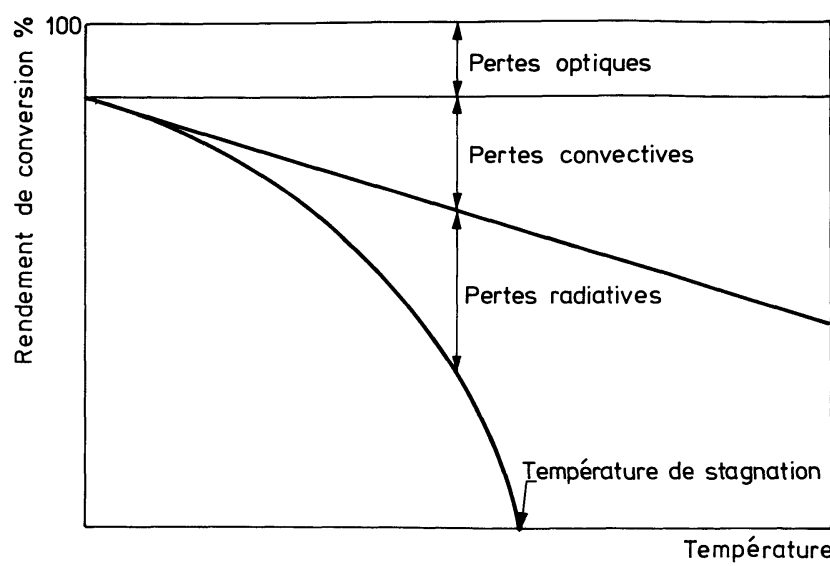

Fig. 2. - Evaluation des pertes d'un capteur en fonction de la température.

[Evaluation of collector losses versus temperature.]

Avant d'entamer une discussion plus détaillée des divers facteurs de pertes, constatons qu'aux basses températures les pertes par convection et conduction, plus particulièrement liées à la conception du capteur, sont prépondérantes. L'effet des pertes radiatives, plus intrinsèquement lié aux propriétés optiques des matériaux de l'absorbeur et du vitrage, devient plus important puis prépondérant aux moyennes puis aux hautes températures. Dans les trois domaines de température, la variation des pertes optiques avec la température peut être considérée comme faible par rapport aux variations des autres pertes.

L'accroissement de la puissance extraite du capteur à une température donnée peut être obtenue dans un premier temps par réduction à une valeur acceptable des pertes thermiques par convection et conduction en agissant sur la géométrie et l'isolation thermique du capteur. Si l'on élimine ce facteur de perte qui, nous l'avons vu, peut être très important en valeur relative, l'expression de la puissance extraite se limite aux deux premiers termes du second membre et l'on peut définir le rendement de conversion du capteur par :

$\eta_{\mathrm{c}}=a-\frac{e}{X E^{\mathrm{s}}} \cdot \sigma\left(T_{\mathrm{a}}^{4}-T_{0}^{4}\right)$

où $X=S_{\mathrm{c}} / S_{\mathrm{a}}$ est le facteur de concentration du flux solaire.

L'accroissement du rendement à une température donnée peut alors être obtenu, soit par action sur la conception du capteur par augmentation du facteur de concentration, moyennant un effort technologique non négligeable et la perte de la quasi-totalité du rayonnement diffus arrivant sur le capteur dès que $X>1$, soit par action sur les propriétés optiques des matériaux composant la couverture et la surface de l'absorbeur de façon à conserver ou augmenter l'absorption de l'énergie solaire incidente et simultanément diminuer ses pertes radiatives à la tempéra- 
ture de fonctionnement. Le rayonnement solaire pouvant être approximé par le rayonnement d'un corps noir à $5900 \mathrm{~K}$ et les températures de conversion étant dans tous les cas inférieures à $1000 \mathrm{~K}$, il y a, ainsi que le montre la figure 3 , une séparation très nette entre la distribution spectrale du rayonnement solaire et celle du rayonnement thermique émis par le

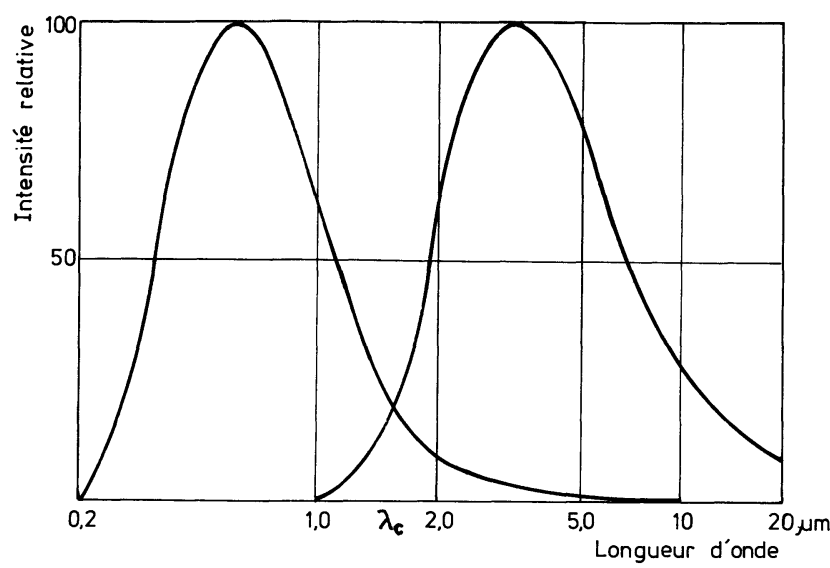

Fig. 3. - Intensités spectrales relatives du rayonnement solaire et du rayonnement émis par un corps noir à $900 \mathrm{~K}$.

[Solar spectral irradiance and black body spectral emittance at $900 \mathrm{~K}$.]

capteur, ce qui permet de définir une longueur d'onde de coupure. La valeur de $\lambda_{c}$ est une fonction croissante du facteur de concentration $X$ et décroissante de la température, comme le montre la figure 4 . Les propriétés optiques demandées aux capteurs seront donc en général bien définies pour une température de fonctionnement $T$ par son absorptivité spectrale $\alpha(\lambda, T)$ et son émissivité spectrale $\varepsilon(\lambda, T)$ intégrées dans tout le demi-espace afin de tenir compte de leur

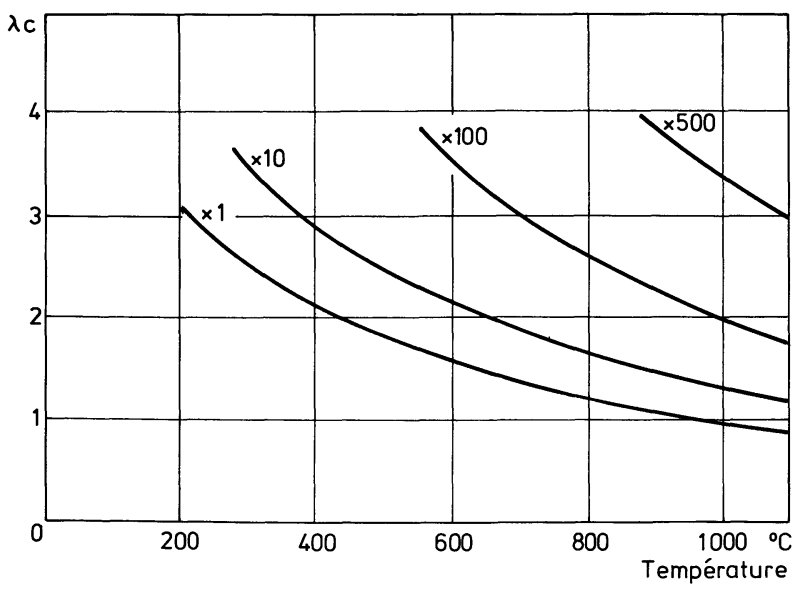

Fig. 4. - Longueur d'onde de coupure optimale $\lambda_{\mathrm{c}}$ pour un corps parfaitement sélectif en fonction de sa température et pour diverses valeurs du facteur de concentration.

[Optimum cut-off wavelength of an ideal selective surface versus temperature, for different concentration factors.] anisotropie. Le capteur sélectif idéal sera alors tel que :

$\left.\begin{array}{lll}\alpha(\lambda, T)=1 & \text { pour } 0<\lambda<\lambda_{\mathrm{c}} \\ \varepsilon(\lambda, T)=0 & \text { pour } & \lambda_{\mathrm{c}}<\lambda\end{array}\right\}$.

Dès 1956, Tabor [1] a recommandé l'utilisation de telles surfaces afin d'accroître le rendement des capteurs solaires. Lorsque le capteur comporte un vitrage disposé en avant de l'absorbeur proprement dit (afin de diminuer les pertes convectives et utiliser l'effet de serre) on peut envisager d'approcher ces conditions idéales de deux manières différentes : la première consiste à équiper le capteur d'un absorbeur sélectif. Dans ce cas, et en supposant le revêtement de l'absorbeur opaque, on cherchera à réaliser :

$\left.\begin{array}{lll}\rho(\lambda, T)=0 & \text { pour } & 0<\lambda<\lambda_{\mathrm{c}} \\ \rho(\lambda, T)=1 & \text { pour } & \lambda_{\mathrm{c}}<\lambda\end{array}\right\}$

où $\rho(\lambda, T)$ est la réflectivité spectrale hémisphérique (intégrée dans $2 \pi \mathrm{sr}$.) du revêtement. La surface de l'absorbeur est alors absorbante ou noire vis-à-vis du rayonnement solaire et réfléchissante ou blanche vis-à-vis du rayonnement infrarouge. Une deuxième solution consiste à utiliser un vitrage sélectif. On cherchera alors à réaliser un traitement du vitrage ayant les propriétés suivantes :

$\left.\begin{array}{lll}\tau(\lambda, T)=1 & \text { pour } & 0<\lambda<\lambda_{\mathrm{c}} \\ \rho(\lambda, T)=1 & \text { pour } & \lambda_{\mathrm{c}}<\lambda\end{array}\right\}$

où $\tau(\lambda, T)=$ transmittivité spectrale hémisphérique (intégrée dans $2 \pi \mathrm{sr}$.) c'est-à-dire transparent pour le rayonnement solaire et réfléchissant vis-à-vis du rayonnement infrarouge émis par l'absorbeur, supposé alors parfaitement absorbant pour le rayonnement solaire. Quel que soit le type de sélectivité utilisé on peut définir des grandeurs $a$ et $e$ intégrées sur tout le spectre et le demi-espace sachant que les quantités monochromatiques directionnelles notées ( $\left(^{\prime}\right)$ sont toujours reliées par :

$\left\{\begin{array}{l}\rho^{\prime}(\lambda, T)+\tau^{\prime}(\lambda, T)+\alpha^{\prime}(\lambda, T)=1 \\ \alpha^{\prime}(\lambda, T)=\varepsilon^{\prime}(\lambda, T) .\end{array}\right.$

L'absorptivité solaire :

$a=\frac{\int_{0}^{\infty} \alpha(\lambda, T) \cdot E^{\mathrm{s}}(\lambda) \cdot \mathrm{d} \lambda}{\int_{0}^{\infty} E^{\mathrm{s}}(\lambda) \cdot \mathrm{d} \lambda}$.

L'émissivité totale :

$e=\frac{\int_{0}^{\infty} \varepsilon(\lambda, T) M^{0}(\lambda, T) \cdot \mathrm{d} \lambda}{\int_{0}^{\infty} M^{0}(\lambda, T) \cdot \mathrm{d} \lambda}$ 
où $E^{\mathrm{s}}(\lambda)$ est l'éclairement solaire monochromatique et $M^{0}(\lambda, T)$ l'émittance spectrale du corps noir à la température $T$.

3. Intérêt des surfaces sélectives. - Si l'on doit choisir entre un capteur solaire avec revêtement sélectif et un capteur n'utilisant pas la sélectivité spectrale, il convient de comparer les rendements de conversion. Les rendements thermoénergétiques i.e., le produit $\mathrm{du}$ rendement de conversion par le rendement de Carnot, doivent aussi être comparés dans le cas où la chaleur extraite est utilisée dans un cycle thermodynamique. Toutes ces comparaisons doivent être faites dans des conditions climatiques données, compte tenu de la conception du capteur. Il convient également de comparer non seulement les rendements instantanés mais leurs valeurs intégrées sur un jour, une semaine, un an. Dans ces conditions, on constate immédiatement qu'il n'y aura pas une solution unique pour obtenir la sélectivité spectrale d'un capteur mais des solutions adaptées à l'application et au site envisagé et prenant en compte les propriétés optiques et le coût du revêtement sélectif. On peut cependant déterminer des domaines de température et de facteur de concentration où l'utilisation de la sélectivité accroît les performances du capteur d'une manière significative.

Comme on a pu le voir figure 2, dans le domaine des basses températures solaires, les pertes thermiques par convection et conduction sont prépondérantes et une réduction même notable des pertes thermiques radiatives ne conduit pas à une augmentation appréciable du rendement. La sélectivité présente donc peu d'intérêt pour des températures de conversion inférieures à $60{ }^{\circ} \mathrm{C}$.

Par contre, dans le domaine des températures moyennes (60 à $\left.150^{\circ} \mathrm{C}\right)$, la sélectivité spectrale peut accroître d'une manière non négligeable le rendement, bien que les pertes convectives et conductives représentent encore une part importante des pertes globales. Dans ce cas, une évaluation précise des nombreux paramètres intervenant dans le rendement est nécessaire pour chiffrer le gain susceptible d'être apporté par la sélectivité. Les résultats obtenus à l'aide de programmes de calcul prenant en compte tous ces paramètres [2] ont permis de déterminer l'influence des propriétés optiques de l'absorbeur et du vitrage sur le rendement pour diverses configurations du capteur (Fig. 5). Le gain est appréciable à toutes les températures et particulièrement au-delà de $60^{\circ} \mathrm{C}$. On remarque aussi que le rendement d'un absorbeur sélectif recouvert d'un simple vitrage est supérieur à celui d'un absorbeur non sélectif équipé d'un double vitrage.

L'utilisation simultanée d'un vitrage sélectif et d'un absorbeur sélectif ou d'un double vitrage non sélectif et d'un absorbeur sélectif ne conduit pas à une amélioration sensible des performances tout en entraînant un surcoût important. Il convient de noter

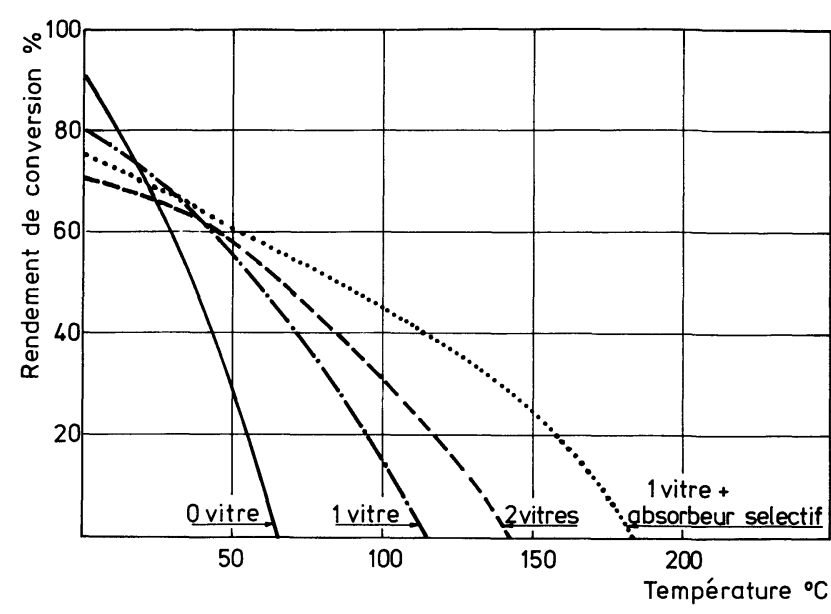

Fig. 5. - Rendement de conversion en fonction de la température pour différents types de capteurs $\left(E^{\mathrm{s}}=1000 \mathrm{Wm}^{-2}\right)$.

[Conversion efficiency versus temperatures for different collector configurations $\left(E^{\mathrm{s}}=1000 \mathrm{Wm}^{-2}\right)$.]

que des températures supérieures à $100^{\circ} \mathrm{C}$ peuvent être obtenues avec des capteurs plans sélectifs avec un rendement de conversion acceptable. Ce type de capteur peut donc être utilisé avec efficacité pour toutes les applications aux moyennes températures : réfrigération, conditionnement d'air, dessalement de l'eau.

Pour les hautes températures solaires, il est nécessaire d'utiliser des systèmes concentrateurs du flux solaire et dans ces conditions on a vu que les pertes par convection et conduction pouvaient être négligées en première approximation par rapport aux pertes radiatives. Sur les figures 6 et 7 on peut comparer les rendements de conversion et thermo-énergétique d'un capteur sélectif $(a=0,9, e=0,1)$ à ceux d'un

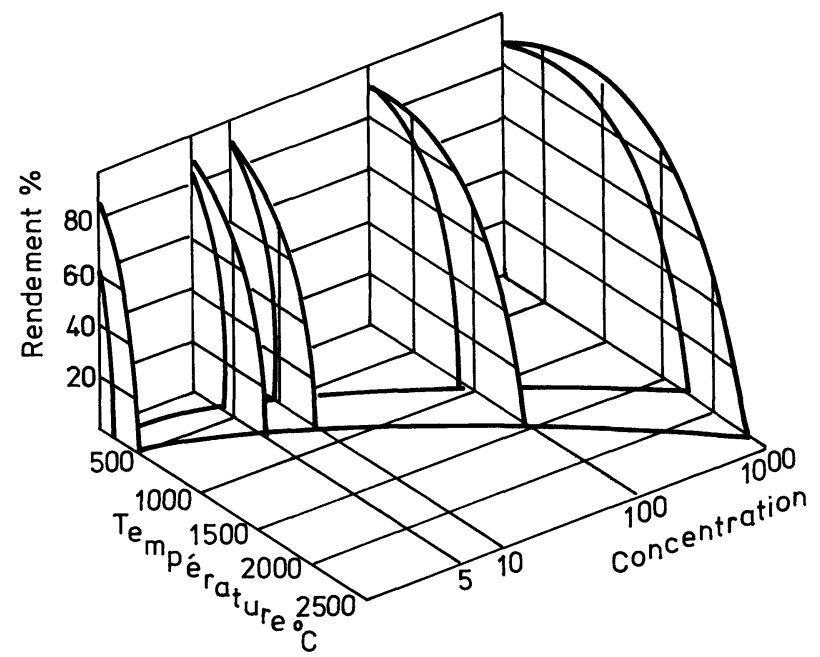

Fig. 6. - Rendement de conversion d'un capteur sélectif ( $a=0,9$, $e=0,1)$ et d'un capteur non sélectif $(a=1, e=1)$ en fonction de la température et pour diverses valeurs du facteur de concentration.

[Conversion efficiency of a selective collector ( $a=0.9, e=0.1$ ) and a non selective collector $(a=1, e=1)$ as a function of temperature, for different values of concentration factor.] 


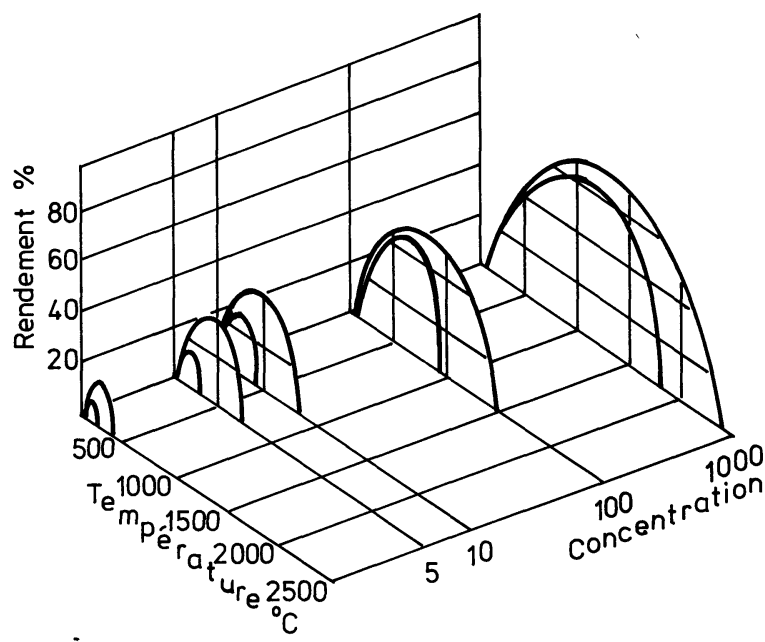

Fig. 7. - Rendement thermo-énergétique d'un capteur sélectif $(a=0,9, e=0,1)$ et d'un capteur non sélectif $(a=1, e=1)$ en fonction de la température et pour diverses valeurs du facteur de concentration.

[Thermoenergetic efficiency of a selective collector $(a=0.9, e=0.1)$ and of a non selective one $(a=1, e=1)$ versus temperature, for different values of the concentration factor.]

capteur se comportant comme un corps noir. On constate que dans tous les cas la sélectivité conduit à une augmentation de rendements mais que ce gain n'est vraiment intéressant que pour des facteurs de concentration inférieurs à 100 et pour des températures inférieures à $1000 \mathrm{~K}$. Sur la figure 8 on a représenté le rendement thermo-énergétique d'un capteur sélectif et d'un capteur non sélectif à la température de conversion optimale en fonction du facteur de concentration. On remarque que le gain introduit par la sélectivité est équivalent à celui produit par une multiplication par $10 \mathrm{du}$ facteur de concentration.

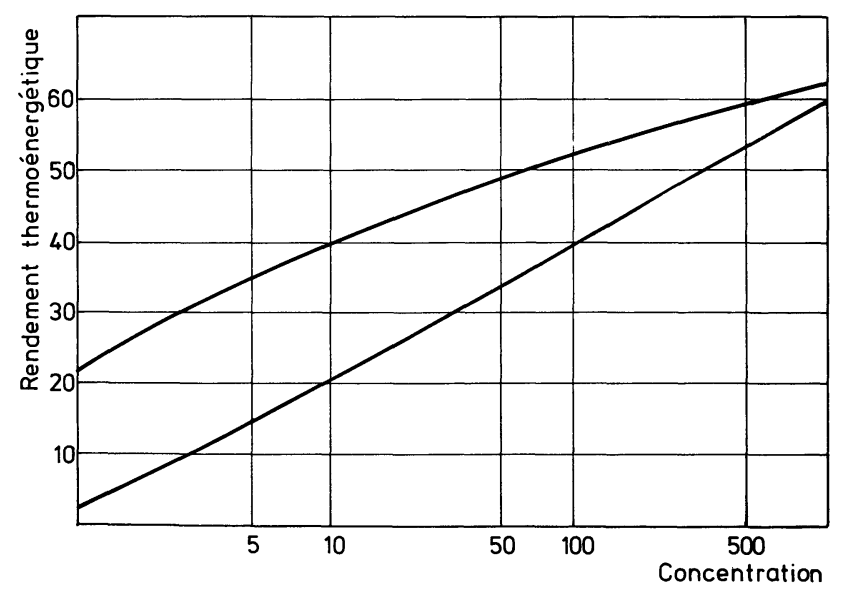

Fig. 8. - Rendement thermo-énergétique d'un capteur sélectif $(a=0,9, e=0,1)$ et d'un capteur non sélectif $(a=1, e=1)$ à la température de conversion optimale en fonction du facteur de concentration.

[Thermoenergetic efficiency of a selective collector $(a=0.9, e=0.1)$ and of a non selective one $(a=1, e=1)$ at the optimum efficiency temperature, versus concentration factor.]
Ceci confirme bien l'intérêt des surfaces sélectives dans la conversion photothermique à haute température.

4. Méthodes d'obtention de surfaces sélectives. Considérant les différents processus physiques mis en jeu, on peut distinguer cinq grandes voies permettant de réaliser la sélectivité spectrale sur le vitrage ou sur la surface de l'absorbeur d'un capteur plan ou non, travaillant avec ou sans concentration du rayonnement solaire $[3,4]$.

4.1 Matériau IntrinsèQue. - Cette solution consiste à utiliser un matériau qui, par ses propriétés intrinsèques (notamment sa structure électronique) présente un comportement optique spectralement sélectif. On connaît des matériaux sélectivement absorbants et transparents. Ainsi, les oxydes diélectriques sont transparents dans le visible et absorbants dans l'infrarouge. A l'opposé, des semi-conducteurs dont le gap est bien choisi, par exemple le silicium, sont transparents dans l'infrarouge et absorbants dans le visible. On connaît aussi le cas de matériaux sélectivement transparents et réflecteurs. Ainsi, les oxydes d'indium et d'étain dopés sont transparents dans le visible et réfléchissants dans l'infrarouge et peuvent par conséquent servir de revêtement sélectif pour le vitrage. La recherche du matériau absorbant pour le spectre solaire et réfléchissant dans l'infrarouge (Fig. 9) est plus délicate et l'étude de la structure électronique des différents types de matériaux permet à l'heure actuelle d'orienter la recherche vers des composés du genre carbures et nitrures de métaux de transition...

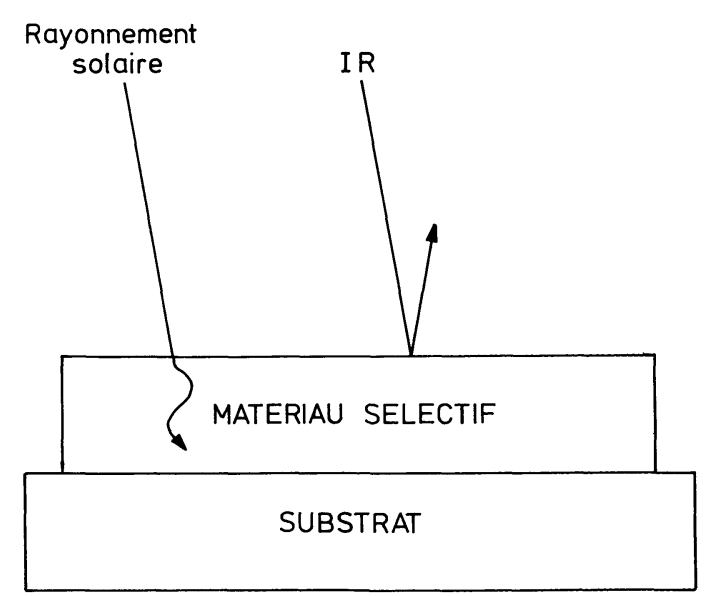

Fig. 9. - Fonctionnement schématique de l'absorbeur sélectif intrinsèque.

[Schematic diagram of the intrinsic selective absorber.]

4.2 Filtre INTERFÉRENTIEL. - La réalisation d'un filtre interférentiel par empilement de plusieurs couches alternées de deux matériaux ayant des indices optiques différents (en général un métal et un diélec- 
trique) et des épaisseurs bien contrôlées peut également conduire à l'effet sélectif recherché (Fig. 10). Ce procédé est malheureusement délicat et coûteux à mettre en œuvre. Des couches à indice continûment variable dans l'épaisseur conduisent sensiblement aux mêmes effets et seraient plus faciles à réaliser.

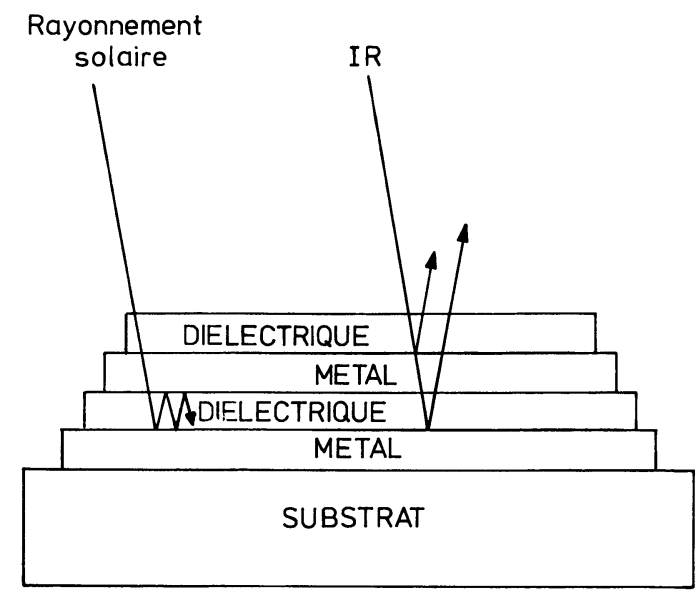

Fig. 10. - Fonctionnement schématique de l'absorbeur sélectif de type interférentiel.

[Schematic diagram of an interference type selective absorber.]

4. 3 L'EMPILEMENT ABSORBEUR + RÉFLECTEUR. Cette troisième voie consiste à réaliser la surface absorbante par le dépôt successif de deux matériaux dont chacun a une fonction optique bien précise. L'absorption du rayonnement solaire est obtenue par l'un, et la réflexion dans l'infrarouge par l'autre. Le couplage absorbeur + réflecteur peut être conçu de deux manières. Dans le premier cas (Fig. 11) on dépose sur un matériau réflecteur dans l'infrarouge (argent, cuivre, aluminium, molybdène...) une couche mince d'un matériau absorbant le rayonnement solaire tout en étant transparent dans l'infrarouge

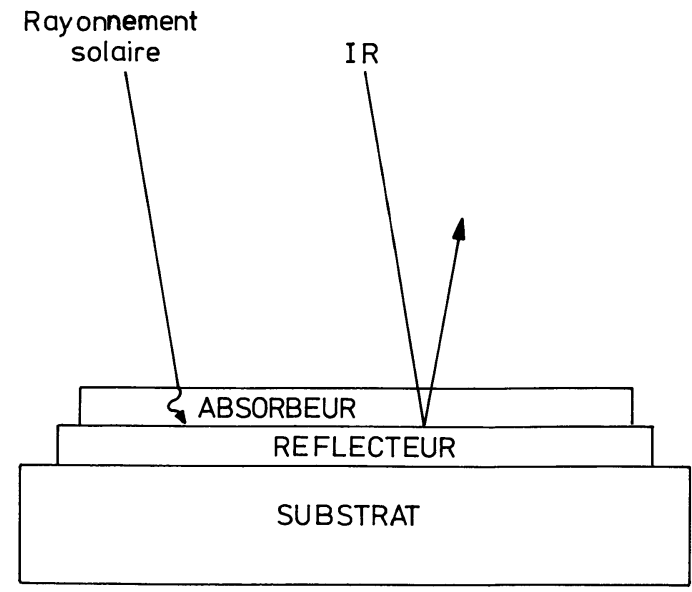

Fig. 11. - Fonctionnement schématique de l'absorbeur sélectif du type réflecteur + absorbeur.

[Schematic diagram of a selective tandem type surface (absorber + reflector).] (silicium, germanium, sulfure de plomb...). Dans le deuxième cas (Fig. 12) on dépose sur un matériau absorbant au moins le rayonnement solaire une couche d'un matériau transparent pour le spectre solaire et réflecteur dans l'infrarouge (oxydes d'étain ou d'indium dopés...).

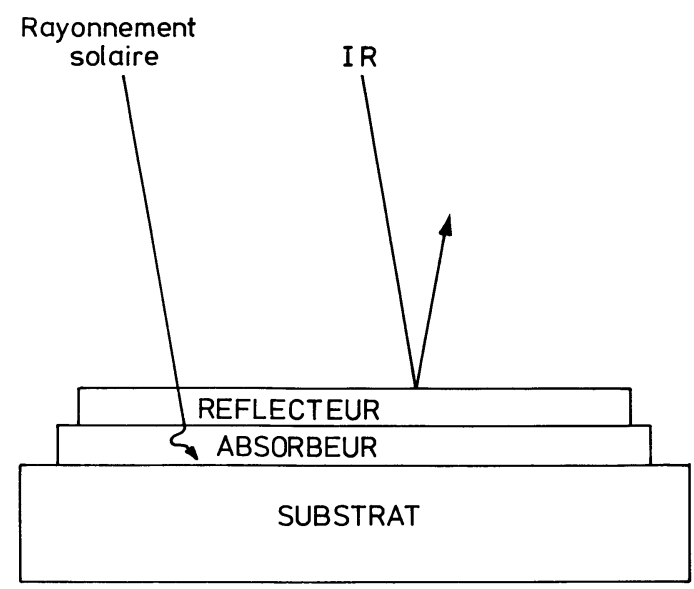

Fig. 12. - Fonctionnement schématique de l'absorbeur sélectif du type réflecteur + absorbeur.

[Schematic diagram of a selective tandem type surface (reflector + absorber).]

4.4 EfFets De Diffusion en volume. - Cette voie utilise l'effet de la diffusion de la lumière dans le volume du revêtement par des inhomogénéités dont les dimensions sont faibles par rapport à la longueur d'onde. Les propriétés optiques d'un tel revêtement sont nettement différentes de celles du matériau homogène par suite des effets de résonance. Les films granulaires de métaux conducteurs et un certain nombre de cermets ayant cette structure présentent une certaine sélectivité spectrale.

4.5 EFFETS DE LA TEXTURe De SURFACE. - Cette dernière voie consiste à utiliser l'influence de la morphologie sur les caractéristiques optiques d'une surface (Fig. 13). En effet, on peut accroître le coeffi-

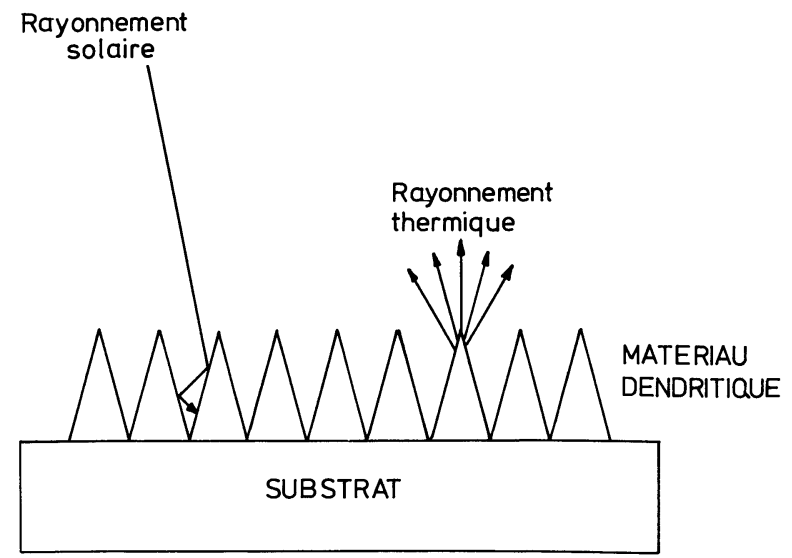

Fig. 13. - Fonctionnement schématique de l'absorbeur sélectif utilisant les effets de texture de la surface.

[Schematic diagram of selective absorber using the effect of surface texture.] 
cient d'absorption solaire d'une surface déjà peu émissive (donc faiblement absorbante) dans l'infrarouge par des réflexions multiples sur des irrégularités de surface de géométrie et de dimensions convenables, sans pour autant accroître notablement l'émissivité infrarouge. Les surfaces corruguées ou dendritiques sont un bon exemple de ce type de surface. Les surfaces sélectives utilisées jusqu'ici pourraient entrer dans plusieurs des catégories définies ci-dessus et il n'est pas toujours facile de déterminer l'effet principal conduisant à la sélectivité.

5. Critères de choix d'une surface sélective. - Malgré la diversité des solutions permettant de réaliser des surfaces optiquement sélectives, le nombre des solutions effectivement utilisables est fort restreint. D'autres contraintes telles que la stabilité des propriétés optiques à long terme et le coût de réalisation doivent en effet être prises en compte. Ce sont ces trois composantes du choix que nous nous proposons d'examiner maintenant.

5.1 Proprí́tés OPtiQues. - Nous avons vu que seules l'absorptivité et l'émissivité spectrales pouvaient rendre compte dans le détail des propriétés optiques d'une surface sélective. Un facteur plus global permettant de comparer, certes moins finement mais plus rapidement des surfaces sélectives entre elles a été proposé comme facteur de mérite. Il s'agit du rapport a/e des valeurs intégrées de l'absorptivité et de l'émissivité. Il est plus judicieux de considérer séparément $a$ et $e$, le rapport $a / e$ ne permettant de déterminer que la température de stagnation du capteur, température atteinte lorsqu'aucune calorie n'en est extraite. Cette température, calculée à partir du bilan énergétique simplifié présenté en (1) s'exprime en négligeant les pertes conductives et convectives par :

$T_{\max }=\left[\frac{a X}{e} \cdot \frac{E^{\mathrm{s}}}{\sigma}+T_{0}^{4}\right]^{1 / 4}$.

Partant de la même forme simplifiée du bilan énergétique on peut également constater qu'une surface sélective dont les caractéristiques optiques totales sont $a$ et $e$ aura un moins bon rendement, dans les mêmes conditions de fonctionnement qu'une surface dont les caractéristiques optiques sont $a+\delta a$ et $e+\delta e$, si la relation suivante est satisfaite :

$\frac{\delta a}{\delta e}>\frac{\sigma\left(T_{\mathrm{a}}^{4}-T_{0}^{4}\right)}{X E^{\mathrm{s}}}$.

Cette inégalité signifie que l'accroissement de l'énergie solaire absorbée est supérieur à l'accroissement des pertes radiatives.

On peut ainsi évaluer l'intérêt de différents absorbeurs sélectifs en tenant compte à la fois des propriétés optiques de l'absorbeur et des conditions de fonctionnement du capteur.
Ainsi, une surface caractérisée par $a=0,9$ et $e=0,2(a / e=4,5)$ se révèle plus efficace qu'une surface caractérisée par $a=0,85$ et $e=0,1$ $(a / e=8,5)$ pour $E^{s}=1000 \mathrm{Wm}^{2}, \quad T_{\mathrm{a}}=60^{\circ} \mathrm{C}$, $T_{0}=25^{\circ} \mathrm{C}$. Par contre, la seconde surface se révèle plus performante que la première si le capteur doit fonctionner dans les mêmes conditions à $120^{\circ} \mathrm{C}$.

Il convient de bien remarquer que la relation (9) ne permet qu'une comparaison grossière entre différentes surfaces sélectives dans la mesure où elle néglige les effets convectifs et conductifs. Elle donne cependant une meilleure idée des performances respectives des surfaces que le rapport $a / e$ souvent abusivement présenté comme une figure de mérite des surfaces sélectives.

5.2 Stabilité PHYSico-ChimiQue. - Il est primordial que les propriétés optiques définies plus haut ne se dégradent pas ou très peu au cours de l'utilisation du capteur. Ceci suppose que la surface sélective supporte la température de fonctionnement, qu'elle ne soit pas affectée par les cycles thermiques d'amplitude et de durée très variables auxquels elle sera soumise et enfin, qu'elle puisse supporter quelques excursions à sa température de stagnation $T_{\max }$ [8] en cas d'arrêt de la circulation du fluide de transfert. Ces contraintes externes peuvent entraîner un certain nombre de détériorations de la surface sélective. Un premier type de détérioration peut être dû à un trop grand écart entre les coefficients de dilatation du substrat (en général cuivre, aluminium ou acier à faible teneur en carbone) et des matériaux composant la surface sélective. Un second type de détérioration est la diffusion d'une couche dans une autre : diffusion chimique ou par les joints de grains. Ce processus conduit dans les deux cas à une détérioration rapide. Ce risque est particulièrement grand dans le cas où la sélectivité est obtenue par effet d'interférences. On peut réduire le risque de diffusion en interposant des barrières de diffusion entre les couches optiquement actives, mais il est bien évident que, plus l'empilement réalisé pour obtenir la sélectivité est complexe, plus grand est le risque de détérioration par interdiffusion.

Enfin, en plus de leur stabilite thermique, les surfaces sélectives doivent être chimiquement inertes, ou pour le moins présenter une cinétique de réaction extrêmement lente afin de résister aux divers types de corrosion auxquels elles peuvent être soumises. $\mathrm{La}$ surface sélective devra donc résister à l'oxydation lorsqu'elle est chauffée et/ou soumise à une irradiation ultraviolette. Le risque d'attaque chimique pendant le stockage avant utilisation ne doit pas non plus être négligé.

5.3 Coût de fabrication. - Il est évident que pour un constructeur de capteurs, une surface sélective est rentable par rapport à un corps noir ou toute autre surface sélective seulement si la quantité de chaleur supplémentaire collectée compense la diffé- 
rence de coût. Cet argument ne devrait cependant pas freiner la recherche au laboratoire de solutions à priori un peu coûteuses soit par les matériaux, soit par les techniques d'élaboration utilisées car l'évolution des techniques et leur transfert à une échelle industrielle modifient les coûts dans des proportions difficilement appréciables. Enfin, compte tenu de la stabilité physicochimique exigée de tout absorbeur, sélectif ou non, il n'est pas évident que l'absorbeur sélectif stable soit beaucoup plus coûteux que son équivalent non sélectif stable. Ce sont cependant les matériaux relativement bon marché associés aux techniques de préparation peu coûteuses (conversion chimique, électrolyse, dépôt chimique en phase vapeur...) qui présentent actuellement le plus d'intérêt.

6. Caractérisation des surfaces sélectives. - Les critères définis au paragraphe 5 suffisent à l'industriel pour choisir une surface sélective. Ils sont nettement insuffisants pour le chercheur qui tente d'améliorer la sélectivité de composants existants ou d'en imaginer d'autres. La caractérisation optique reste, bien sûr, essentielle, la détermination de la composition chimique, de la structure en volume et en surface ne l'est pas moins.

6.1 Caractérisation optiQue. - Pour des échantillons non diffusants, elle suppose la mesure de la réflectivité bi-directionnelle et éventuellement de la transmittivité (dans le cas de traitement du vitrage) dans une gamme spectrale de 0,3 à environ 15 ou $20 \mu \mathrm{m}$ (suivant les températures d'utilisation du matériau) en fonction de l'angle d'incidence et de la polarisation du rayonnement incident. C'est notamment à partir de ces quantités monochromatiques directionnelles que l'on peut séparer les contributions des rayonnements direct et diffus tombant sur la surface ou encore calculer les variations de l'absorption solaire au cours d'une journée lorsque l'angle d'incidence du rayonnement direct varie sur le capteur. C'est enfin à partir de ces données et par intégrations successives sur l'espace et les longueurs d'onde que l'on remonte aux quantités a et e auxquelles on peut, bien sûr, accéder directement par mesures calorimétriques. Dans le cas d'échantillons diffusants on mesure la réflectivité directionnelle hémisphérique. Pour ces études, deux sphères intégrantes couvrant le spectre visible et l'infrarouge jusqu'à $14 \mu \mathrm{m}$ ont été utilisées. Ces sphères, mises au point dans nos laboratoires, (l'une recouverte de peinture blanche diffusante, l'autre de microbilles de verre recouvertes d'une couche d'or) jouent le rôle de source et de porte-échantillon. Elles permettent de mesurer la réflectivité directionnelle pour un flux incident isotrope, ce qui, en vertu du principe de réciprocité équivaut à la mesure de la réflectivité directionnelle hémisphérique.

6.2 Autres Caractérisations. - La composition est déterminée suivant les composés par analyse chimique ou diffraction des rayons $\mathrm{X}$ ou d'électrons ou, en dernier ressort et plus qualitativement, par ESCA.

La texture de surface peut être déterminée par microscopie classique, ou mieux, par microscopie électronique à balayage. On peut également faire des mesures de rugosité. La diffraction de rayons $X$ ou d'électrons après attaque chimique du substrat ou amincissement ionique permettent d'obtenir des informations sur la structure en volume.

Les épaisseurs enfin sont déterminées soit à l'aide d'un Talystep, soit par méthodes interférométriques (interférences de rayons $\mathrm{X}$ en incidence rasante dites franges de Kiessing pour les faibles épaisseurs ou méthode de Tolansky pour les épaisseurs plus fortes).

7. Exemples de surfaces sélectives. - Il serait vain de vouloir présenter une liste exhaustive de toutes les surfaces sélectives étudiées jusqu'ici. On présente rapidement ici un certain nombre de surfaces sélectives utilisables à moyennes et hautes températures et on détaille le cas des composés élaborés et étudiés dans nos laboratoires car ils semblent constituer des solutions intéressantes dans chacun de ces domaines de température.

7.1 SURFACES SÉLECTIVES POUR LA CONVERSION « MOYENNES TEMPÉRATURES » $\left(60^{\circ} \mathrm{C}<\mathrm{T}<150^{\circ} \mathrm{C}\right)$. Exemple du chrome noir. - Compte tenu des différents paramètres et critères envisagés plus haut, un absorbeur sélectif travaillant dans le domaine des moyennes températures devra remplir les conditions suivantes :

- l'absorptivité solaire " $a$ » doit être aussi voisine que possible de l'unité et en tout cas supérieure à 0,9 ;

- l'émissivité « $e$ » doit être aussi faible que possible mais des valeurs de 0,1 ou 0,2 sont encore acceptables ;

- la stabilité thermique et la résistance à la corrosion doivent assurer à la surface une durée de vie d'au moins 10 ans ;

- le procédé de préparation doit pouvoir être adapté à la production de masse des surfaces de grandes dimensions (environ $2 \mathrm{~m}^{2}$ );

- le surcoût d'une telle surface doit être acceptable compte tenu du gain apporté aux performances du capteur.

C'est pour toutes ces raisons qu'un grand nombre d'études ont été menées sur des surfaces absorbantes sélectives produites par des procédés dérivés de ceux déjà couramment utilisés par l'industrie pour réaliser des traitements décoratifs noirs ou anti-réfléchissants dans le visible. Le choix du traitement dépend dans une large mesure de la nature du matériau constituant le substrat de l'absorbeur. Les matériaux les plus couramment utilisés sont l'acier doux, le cuivre et l'aluminium. Le tableau I rend compte des résultats les plus intéressants obtenus jusqu'à présent. Les 
Tableau I. - Surfaces sélectives pour la conversion photothermique à "basse » et "moyenne » températures.

\begin{tabular}{llcccc}
\multicolumn{1}{c}{ Substrat } & Traitement & Dépôt & $a$ & $e$ & Réf. \\
Cuivre & - & - & - & - & - \\
Cuivre & conversion & $\mathrm{CuO}^{-} \mathrm{Cu}_{2} \mathrm{O}$ & 0,85 & 0,1 & {$[1]$} \\
Aluminium & coctrolyse & $\mathrm{CrO}_{x}\left(^{*}\right)$ & 0,97 & 0,09 & {$[12]$} \\
Acier & conversion & $\mathrm{CuO}^{-} \mathrm{CuO}_{2}$ & 0,93 & 0,11 & {$[7]$} \\
Acier & électrolyse & $\mathrm{Fe}_{3} \mathrm{O}_{4}$ & 0,85 & 0,12 & {$[8]$} \\
Acier & électrolyse & $\mathrm{NiS}^{2} \mathrm{ZnS}$ & 0,95 & 0,07 & {$[9-10]$} \\
& & $\mathrm{CrO}_{x}\left(^{*}\right)$ & 0,95 & 0,13 & {$[11]$}
\end{tabular}

(*) Nos échantillons.

surfaces de chrome noir qui paraissent les plus prometteuses ont été systématiquement préparées et étudiées dans nos laboratoires.

Le dépôt est effectué par électrolyse d'un bain à base d'acide chromique exempt d'ions sulfate. On a utilisé comme substrats l'acier doux nickelé et le cuivre nickelé ou non. En agissant sur les divers paramètres de préparation : composition du bain, temps de dépôt, densité de courant, état de surface du substrat, on arrive à optimiser les valeurs de a et de e de la surface sélective ainsi produite. Tous les autres paramètres étant figés, on observe l'existence d'une durée d'électrolyse optimale au bout de laquelle l'absorptivité solaire " $a$ » se stabilise (alors qu'elle croissait auparavant) tandis que l'émissivité infrarouge " $e$ » commence à croître (alors qu'elle était relativement constante auparavant). Les études de diffraction d'électrons et de rayons $\mathbf{X}$ faites sur un échantillon préparé dans ces conditions montrent l'existence dans le volume du matériau déposé de petites particules de chrome métallique de l'ordre de $100 \AA$ à $200 \AA \AA$ de diamètre dispersées dans une matrice amorphe d'oxydes de chrome $\mathrm{CrO}_{x}$. L'analyse par spectrométrie de photoélectrons (ESCA) révèle que le rapport chrome/oxygène croît au fur et à mesure que l'on se rapproche du substrat, suggérant que le taux de remplissage du chrome dans l'oxyde va croissant dans le même sens. De plus, les revêtements réalisés sur acier reproduisent en surface les rugosités $\mathrm{du}$ substrat dont les dimensions caractéristiques (hauteur et période) sont de l'ordre de $2 \mu \mathrm{m}$ et diffusent fortement la lumière (Fig. 14). Les revêtements réalisés sur cuivre ne présentent pas cette rugosité importante et sont pratiquement non diffusants (Fig. 14). Il subsiste cependant, dans les deux cas, en surface, une microrugosité de l'ordre de $1000 \AA$ qui n'entraîne donc pas de diffusion incohérente notable.

L'explication que l'on peut donner à l'heure actuelle de la sélectivité de tels revêtements relève à la fois du couplage absorbeur + réflecteur et des effets de diffusion résonnante; les effets de diffusion en surface lorsque le matériau est rugueux viennent bien sûr s'ajouter. Les premiers essais de modélisation de la réflectivité qui ont été entrepris sur ces revêtements montrent que le milieu diélectrique non homogène

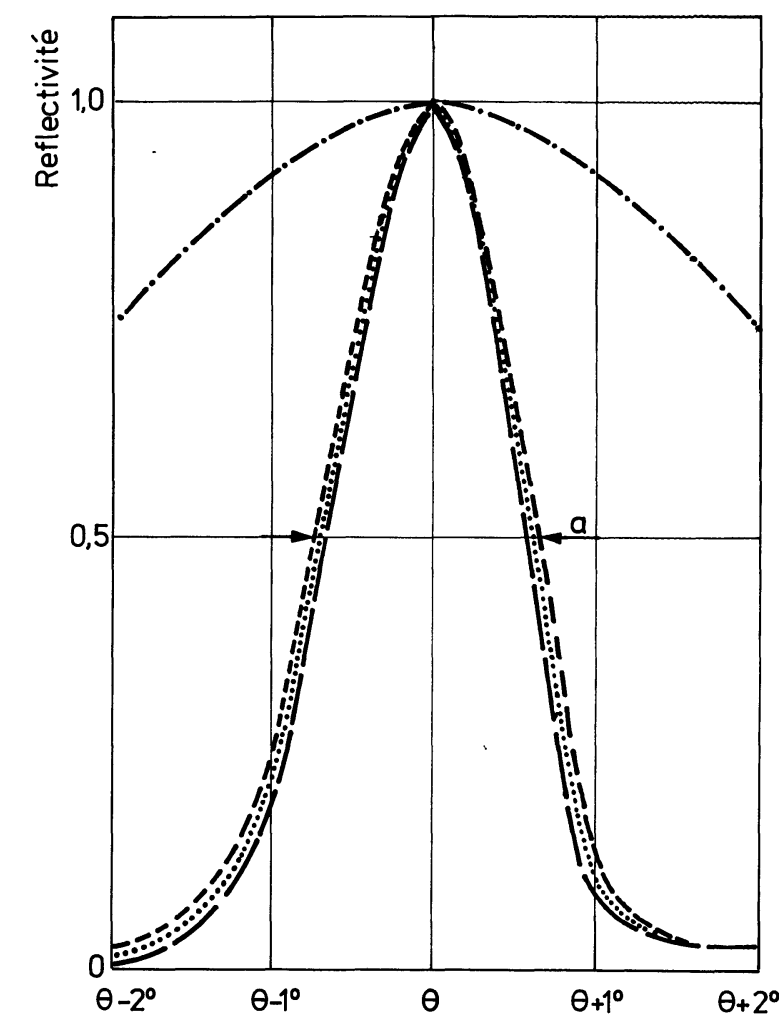

Fig. 14. - Dispersion de la réflectivité bi-directionnelle autour de la direction spéculaire du faisceau réfléchi $\left(\theta=15^{\circ}\right)$ :

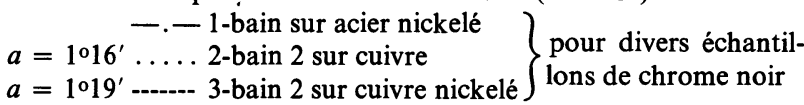

$a=11^{\circ} 19^{\prime}---3$-bain 2 sur cuivre nickelé
$a=1^{\circ} 13^{\prime}-1$-pour un échantillon spéculaire d'or (dans cas l'étalement de la courbe ne traduit pas l'effet de la dispersion mais simplement le facteur d'appareil du système de mesure).

[Angular dispersion of bi-directional reflectivity around the spectral reflectance angle $\left(\theta=15^{\circ}\right)$.

-. 1-bath 2 on nickel steel $\}$ for various chromium $\left.\begin{array}{l}a=1^{\circ} 16^{\prime} \ldots \ldots 2 \text {-bath } 2 \text { on copper } \\ a=1^{\circ} 19^{\prime} \ldots \text {-...- 3-bath } 2 \text { on copper-nickel }\end{array}\right\} \begin{aligned} & \text { for various ch } \\ & \text { black samples }\end{aligned}$

$a=1^{\circ} 13^{\prime}--4$-for a smooth gold sample ( $a=$ instrument function).]

se comporte optiquement comme un semi-conducteur avec un gap d'environ $0,4 \mathrm{eV}$, i.e. transparent dans l'infrarouge et absorbant dans le spectre solaire. Les effets de diffusion résonnante qui interviennent dans cette matrice d'oxydes avec grains de chrome sont modélisés à partir de la théorie de MaxwellGarnett [5] modifiée par Cohen, Cody, Coutts et Abeles [6] afin de prendre en compte des taux de remplissage en chrome élevés au voisinage du substrat. L'effet anti-reflet de surface paraît devoir être attribué à une variation continue de l'indice de l'oxyde dans l'épaisseur du matériau liée à la rugosité résiduelle d'environ $1000 \AA$ à la surface de l'oxyde. La couche de nickel ou le cuivre massif joue évidemment le rôle de réflecteur infrarouge.

Sur la figure 15 ont été représentées la réflectivité hémisphérique pour un flux incident quasi normal 


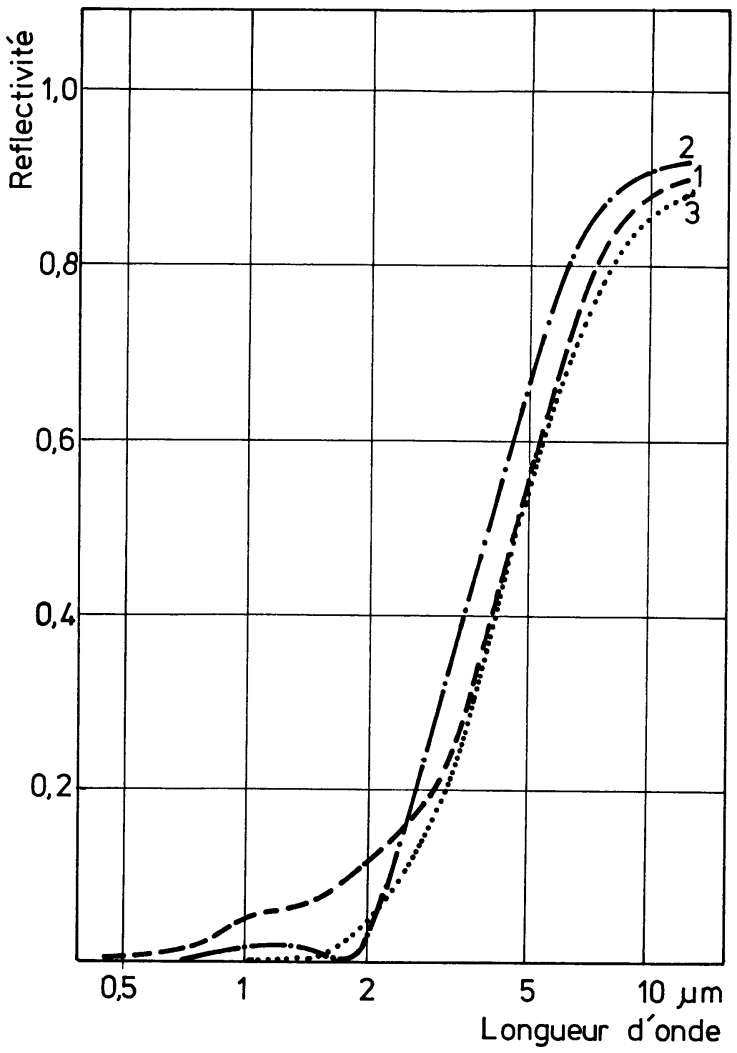

Fig. 15. - Réflectivité hémisphérique d'une surface de chrome noir (bain 1) déposée sur acier rugueux nickelé (-----) et réflectivité spéculaire de 2 surfaces de chrome noir (bain 2) déposé sur cuivre (échantillon $2-.-$ ) et sur cuivre nickelé (échantillon $3 \ldots$..) pour une incidence quasi normale du rayonnement.

[Hemispherical reflectivity of a black chromium surface (bath 2) deposited on rough/nickeled steel (-----) and specular reflectivity of two black chromium surfaces (bath 2) deposited on copper (sample 2 -.-) and on nickeled copper (sample 3 ....) for quasi normal incident radiation.]

d'une surface de chrome noir déposé sur acier rugueux recouvert de $10 \mu \mathrm{m}$ de nickel (échantillon 1 diffusant) ainsi que la réflectivité spéculaire pour un flux incident quasi normal de deux surfaces de chrome noir déposées l'une sur cuivre, l'autre sur cuivre recouvert de $10 \mu \mathrm{m}$ de nickel avec des conditions opératoires différentes (échantillons 2 et 3 non diffusants). La fréquence de coupure étant de l'ordre de $3 \mu \mathrm{m}$ ce revêtement convient bien à des applications basses températures

Tableau II. - Performances de nos 3 échantillons de chrome noir.

No

$\begin{array}{lccc}\text { échantillon } & 1 & 2 & 3 \\ \begin{array}{l}- \\ \text { bain }\end{array} & 1 & \frac{2}{2} & \frac{2}{2} \\ \text { substrat } & \text { acier }+\mathrm{Ni} & \mathrm{Cu}+\mathrm{Ni} & \mathrm{Cu} \\ e \text { (ambiante) } & 0,13_{4} & 0,14_{0} & 0,09_{5} \\ \begin{array}{c}a \text { (masse } \\ \text { d'air } 1)\end{array} & 0,95_{5} & 0,96_{8} & 0,97\end{array}$

et moyennes températuires. On observe une nette amélioration de l'absorptivité solaire au passage du premier bain (échantillon 1) au deuxième (échantillons 2 et 3) mais surtout dans le cas des deux derniers échantillons on sépare bien le comportement du cuivre (échantillon 2) plus réfléchissant dans l'infrarouge de celui du nickel (échantillon 3). Le tableau II rend compte des performances des 3 échantillons étudiés.

A titre de comparaison, la figure 16 présente les rendements réels de conversion d'un capteur équipé de l'absorbeur sélectif correspondant à l'échantillon 1 et d'un capteur comportant un absorbeur peint en noir dans les mêmes conditions de fonctionnement. Le traitement au chrome noir, mis au point au CENG, a été développé à l'échelle industrielle sur des panneaux de $2 \mathrm{~m}^{2}$ et semble donc constituer à l'heure actuelle l'une des meilleures solutions pour réaliser des absorbeurs sélectifs pour les applications à basses et moyennes températures.

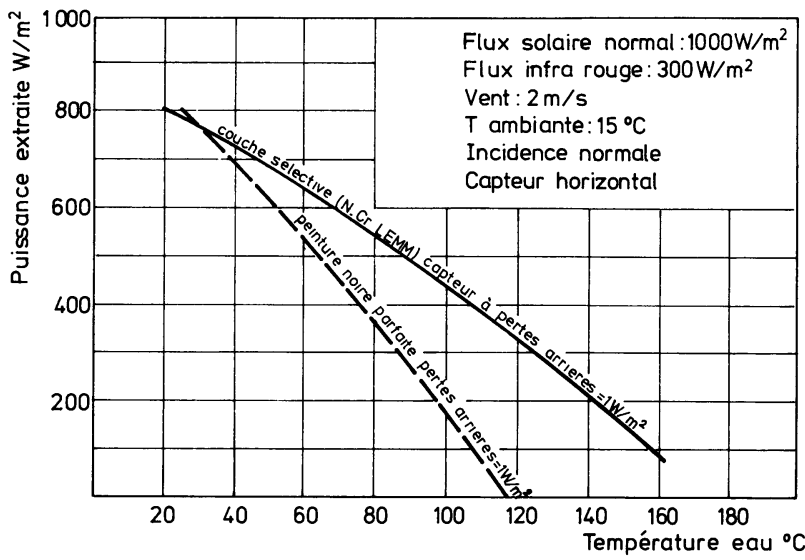

Fig. 16. - Rendements réels de conversion d'un capteur sélectif (échantillon 1 de chrome noir) et d'un capteur équipé d'un absorbeur recouvert de peinture noire.

[Real conversion efficiencies of a selective collector (black chromium sample $\mathrm{n}^{\circ}$ ) and of a non selective one (black paint).]

7.2 SURFACES SÉLECTIVES POUR LES « HAUTES TEMPÉRATURES ". - - Lorsque les températures de conversion sont élevées, les caractéristiques optiques de la surface absorbante sélective ont un effet encore plus marqué sur les performances du capteur, les pertes radiatives prenant une importance considérable (Fig. 2). Seuls les capteurs à concentration permettent d'obtenir des températures de conversion largement supérieures à $150^{\circ} \mathrm{C}$. Les propriétés optiques, thermiques et chimiques des surfaces absorbantes utilisées dans ce cas sont notablement différentes de celles exigées des absorbeurs plans :

- l'absorptivité solaire " $a$ » doit être au moins de 0,85 à la température de fonctionnement,

- l'émissivité infrarouge " $e$ " ne doit pas être supérieure à 0,1 même à la température de fonctionnement, 
- la surface doit être thermiquement et chimiquement stable sur une très longue période, à la température de fonctionnement et sous irradiation ultraviolette, et sur un temps relativement court, à la température de stagnation. Ces dernières conditions sont certainement les plus contraignantes.

Etant donné que le coût de l'absorbeur d'un capteur à concentration ne représente qu'une part relativement faible du coût total, la sélectivité peut, dans ce cas, être réalisée avec des matériaux et des procédés plus onéreux que ceux retenus pour les absorbeurs plans. Il n'est donc pas impensable d'envisager l'emploi de techniques de dépôt sous vide ou de dépôt chimique en phase vapeur pour réaliser des surfaces ayant les caractéristiques optiques requises. Les meilleurs résultats obtenus à ce jour sont rassemblés dans le tableau III. Ces résultats ont été obtenus à l'échelle du laboratoire et il est douteux que les procédés utilisés puissent tous être développés à l'échelle industrielle. C'est notamment le cas des procédés utilisant le CVD. L'empilement silicium sur argent proposé par Seraphin [4] entre dans cette catégorie. Cette surface possède des propriétés optiques convenables et une bonne stabilité thermique aux températures inférieures à $500^{\circ} \mathrm{C}$. Il est clair que plus le nombre de couches est élevé, plus le risque de détérioration par interdiffusion est grand et plus la réalisation de l'empilement est délicate. Les surfaces sélectives composées d'une ou deux couches chimiquement compatibles entre elles et avec le substrat semblent plus réalistes dans la mesure où le risque de détérioration sous l'effet de température est réduit et où le nombre d'opérations nécessaires à leur élaboration est limité.

Tableau III. - Surfaces sélectives pour la conversion photothermique à " haute température ".

$\begin{array}{llcccc}\text { Substrat } & \text { Traitement } & \text { Dépôt } & a & e & \text { Réf. } \\ - & - & - & - & - & - \\ \text { Molybdène } & \text { évaporat. } & \left(\mathrm{Al}_{2} \mathrm{O}_{3}-\mathrm{Mo}\right)_{x} & 0,91 & 0,16 & {[13]} \\ \text { Aluminium } & \text { évaporat. } & \mathrm{PbS} & 0,90 & 0,04 & {[14]} \\ \text { Acier } & \text { pulv. cath. } & \mathrm{Ag} / \mathrm{TiN} & 0,88 & 0,08 & {[17]} \\ \text { Acier } & \text { pulv. cath. } & \mathrm{Ag} / \mathrm{ZrO}_{x} \mathrm{~N}_{y} & 0,93 & 0,28 & {[18]} \\ \text { Acier } & \text { évaporat. } & \mathrm{Ag} / \mathrm{CrO}_{x} & 0,87 & 0,05 & {[19]} \\ \text { Acier } & \text { DCPV }\left(^{*}\right) & \mathrm{Ag} / \mathrm{Si}^{2} & 0,75 & 0,08 & {[4]} \\ \text { Cuivre } & \text { pulv. cath. } & \mathrm{Fe}_{3} \mathrm{C} & 0,9 & 0,04 & {[16]}\end{array}$

$\left(^{*}\right)$ Dépôts chimiques en phase vapeur.

Deux types de surfaces sélectives remplissent ces conditions : en premier lieu celles composées de deux surfaces actives, tel le tandem réalisé par Harding [16] qui a déposé sur un substrat réflecteur en cuivre un absorbeur composé de carbure de fer, chrome, nickel, tantale, molybdène et tungstène par pulvérisation cathodique réactive. Cette surface sélective est stable jusqu'à $500^{\circ} \mathrm{C}$ et ses propriétés optiques sont acceptables pour la conversion à haute température.
En second lieu, les matériaux intrinsèquement sélectifs restent à découvrir mais on commence à approcher les propriétés grâce aux carbures et nitrures des métaux de transition. Les premiers résultats obtenus ne figurent pas dans le tableau III dans la mesure où leur absorptivité solaire est encore trop faible. Ces composés possèdent une remarquable stabilité des propriétés optiques à haute température, ainsi que le montrent par exemple les études de Seraphin [4] sur le carbure de hafnium. L'intérêt des carbures et nitrures de titane préparés et étudiés dans nos laboratoires réside dans le fait que ces composés existent dans un large domaine de concentration, ce qui permet, dans une certaine mesure, d'ajuster la fréquence de coupure $\lambda_{\mathrm{c}}$, l'absorptivité solaire " $a$ » et l'émissivité infrarouge " $e$ » en jouant sur leur composition. Les composés $\mathrm{TiN}_{x}$ et $\mathrm{TiC}_{x}$ sont préparés par pulvérisation réactive en présence de $\mathrm{N}_{2}$ ou $\mathrm{CH}_{4}$. Les paramètres essentiels du dépôt sont la pression partielle du gaz, la vitesse d'évaporation et la température du substrat. Le profil le plus intéressant (Fig. 17) est celui de TiN qui présente notamment une faible émissivité infrarouge. Cependant, ses qualités d'absorption sont insuffisantes et sa fréquence de coupure $\lambda_{\mathrm{c}}<1 \mu \mathrm{m}$ trop faible même pour les applications à hautes températures. Les échantillons de $\mathrm{TiC}$ (Fig. 18) présentent quant à eux une transition visible-infrarouge trop peu accentuée. La transition est plus nette pour les écarts à la stochiométrie les plus élevés, mais on perd alors sur la réflectivité infrarouge. L'échantillon produit avec la plus faible pression de $\mathrm{CH}_{4}$ a un comportement analogue à celui du titane pur.

$\mathrm{La}$ réalisation de structures dendritiques de l'ordre de $1 \mu \mathrm{m}$ (période) à la surface de ces composés permet de modifier considérablement leur profil spectral

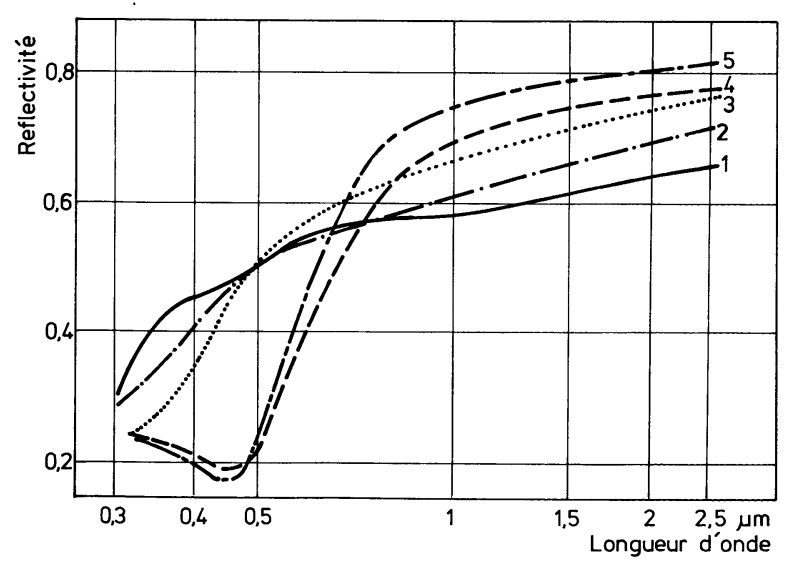

Fig. 17. - Réflectivité spéculaire de divers échantillons $\mathrm{TiN}_{x}$ éclairés sous incidence quasi normale : pression partielle d'azote pendant la pulvérisation; -0 torr (1); - . - 4 $\times 10^{-4}$ torr (2); .... $5 \times 10^{-4}$ torr (3) ; ---- 5,5 $\times 10^{-4}$ torr (4) $;-\times-10^{-3}$ torr (5).

[Specular reflectivity of various samples $\mathrm{TiN}_{x}$ under quasi normal incidente light : partial pressure of nitrogen during evaporation; -0 torr (1); - . $-4 \times 10^{-4}$ torr (2); ... $5 \times 10^{-4}$ torr (3); $---5 \times 5 \times 10^{-4}$ torr $(4) ;-\times-10^{-3}$ torr (5).] 


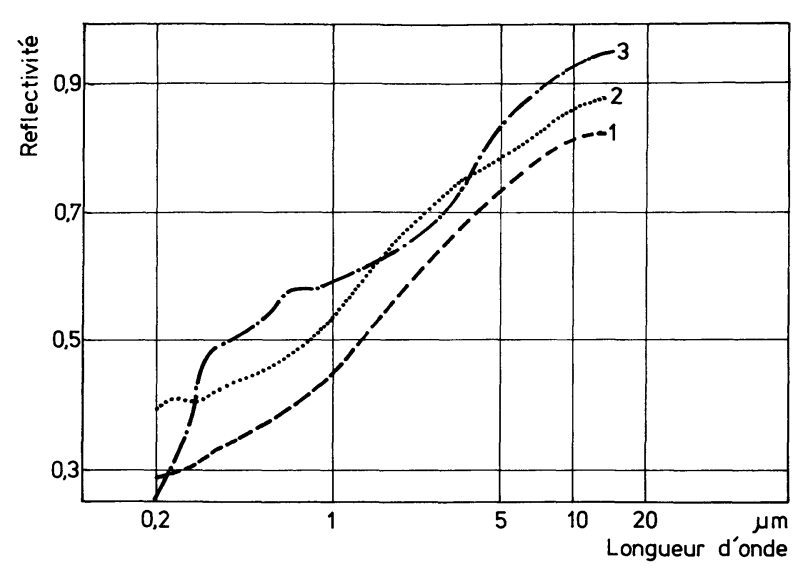

Fig. 18. - Réflectivité spéculaire de divers échantillons de $\mathrm{TiC}_{x}$ éclairés sous incidence quasi normale : pression partielle de $\mathrm{CH}_{4}$ pendant la pulvérisation ; ----- $8 \times 10^{-4}$ torr ; .... $4 \times 10^{-4}$ torr; - $-2 \times 10^{-4}$ torr.

[Specular reflectivity of various samples of $\mathrm{TiC}_{x}$ under quasi normal incident light : partial pressure of $\mathbf{C H}_{4}$ during evaporation; ---- $8 \times 10^{-4}$ torr ; $\ldots . .4 \times 10^{-4}$ torr $;-.-2 \times 10^{-4}$ torr.]

comme le montrent les variations de la réflectivité hémisphérique représentées sur la figure 19. Dans ce cas de TiN dendritique proche de la stœchiométrie, la fréquence de coupure a été considérablement déplacée jusque vers $10 \mu \mathrm{m}$. L'absorptivité solaire a été quelque peu améliorées hormis un pic vers $0,65 \mu \mathrm{m}$. Ces premières tentatives demandent à être poursuivies

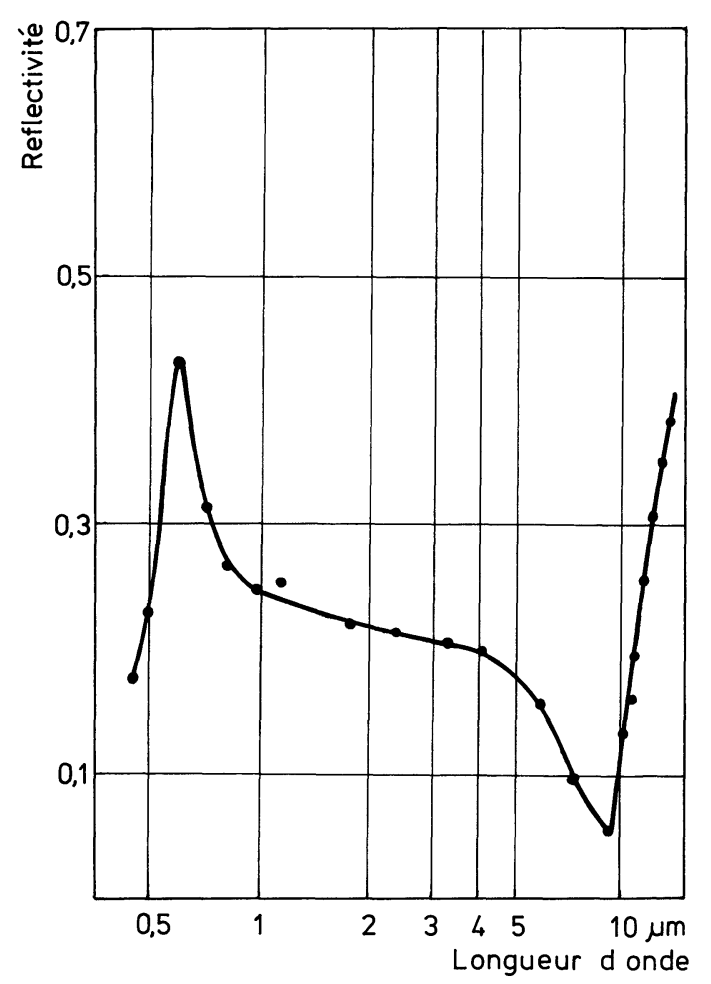

Fig. 19. - Réflectivité hémisphérique d'un échantillon de $\mathrm{TiN}_{x}$ dendritique (proche de la stæchiométrie) éclairé sous incidence quasi normale.

[Hemispherical reflectivity of a dendritic $\operatorname{TiN}_{x}$ sample $(x \simeq 1)$ under near normal incidence.] dans la mesure où l'on espère, en contrôlant les dimensions des dendrites, pouvoir maîtriser le déplacement de $\lambda_{\mathrm{c}}$ et aussi l'absorptivité solaire de la surface.

7.3 SÉleCtivité SPECTRALE SUR LE VITRAGE DE COUVERTURE. - Il existe en principe différentes manières de réaliser des verres sélectifs i.e. transparents dans le spectre solaire et réfléchissants dans l'infrarouge :

- par dépôt de couches minces métalliques jouant le rôle de filtre. L'épaisseur déposée est critique vis-àvis de la transmission solaire. De plus, les pertes par réflexion sur ces couches étant importantes, il est nécessaire de leur appliquer un traitement antiréfléchissant ce qui conduit au minimum à un empilement diélectrique-métal-diélectrique. Un certain nombre de verres sélectifs ont été ainsi réalisés avec, comme diélectrique : le sulfure de zinc [20], l'oxyde de bismuth [21] ou l'oxyde de titane [22-23] et comme métal : l'or, l'argent ou le cuivre. Dans le meilleur des cas [22] on ne dépasse pas une transmittivité solaire de 0,72 , ce qui est trop faible pour l'utilisation dans la conversion photo-thermique mais parfaitement adapté pour les vitrages destinés à l'isolation thermique des bâtiments (avec $e=0,05$ ) ;

- par dépôts de microgrilles [24-26] dont la maille est de l'ordre de grandeur de la longueur d'onde de coupure recherchée. Ces systèmes se comportent vis-à-vis du rayonnement lumineux incident comme une antenne vis-à-vis du rayonnement électro-magnétique. Transparents aux longueurs d'onde inférieures aux dimensions du maillage ils seront, par contre, réfléchissants pour les longueurs d'onde plus élevées. Là encore, les médiocres performances en transmission de ces systèmes les écartent des applications à la conversion ;

- par dépôt d'oxydes transparents. On peut en effet obtenir l'effet sélectif recherché par dépôt sur le verre d'une couche d'un matériau dont la propriété intrinsèque est d'être simultanément transparent pour le spectre solaire et réflecteur pour le rayonnement infrarouge. De tels matériaux existent. Ce sont les oxydes semi-conducteurs tels que $\mathrm{SnO}_{2}, \mathrm{In}_{2} \mathrm{O}_{3}, \mathrm{CdO}$ ou des mélanges binaires de ces oxydes. Ces oxydes se comportent comme des semi-conducteurs de type « $\mathrm{n}$ ", la conduction résultant de la présence de charges négatives dues aux lacunes d'oxygène. Pour présenter une réflexion à caractère métallique dans l'infrarouge il est nécessaire que ces matériaux possèdent une résistivité électrique faible et, par conséquent, une densité de porteurs de charge libres élevée. On montre que pour avoir une réflexion dans l'infrarouge supérieure à $70 \%$ il faut que la densité en porteurs de charge libres soit supérieure à $10^{20} \mathrm{~cm}^{-3}$. Une telle densité peut être obtenue soit par un taux élevé de lacunes d'oxygène et par suite un écart important à la stœchiométrie, soit par dopage de l'oxyde par des éléments ayant une valence supérieure à celle du métal ou inférieure à celle de l'oxygène. 
Du fait de ses propriétés optiques et électriques particulières ce type de matériau trouve des applications dans des domaines très variés et il fait donc l'objet d'un grand nombre de travaux dont Vossen [27] rend compte dans un article de revue très récent. Des oxydes transparents conducteurs ont été obtenus par dépôt chimique en phase vapeur [28-32] par évaporation réactive et par pulvérisation cathodique [33-34]. Le tableau IV rend compte des résultats les plus significatifs obtenus.

Tableau IV. - Caractéristiques optiques des oxydes transparents conducteurs.

\begin{tabular}{clrcccc} 
& \multicolumn{1}{c}{ Procédé } & \multicolumn{3}{c}{ Propriétés optiques } & Réfé- \\
Matériau & d'élaboration & $R\left({ }^{1}\right) \%$ & $T\left(^{2}\right) \%$ & $n$ & rences \\
- & -- & - & - & - & - \\
$\mathrm{SnO}_{2}$ & Pyrolyse & - & $90-95$ & $1,75-2,20$ & {$[28]$} \\
$\mathrm{SnO}_{2} / \mathrm{Sb}$ & Pyrolyse & & $85-91$ & 1,89 & {$[29]$} \\
$\mathrm{In}_{2} \mathrm{O}_{3}$ & Pyrolyse & - & $<90$ & - & {$[30]$} \\
$\mathrm{In}_{2} \mathrm{O}_{3} / \mathrm{Sn}$ & Pyrolyse & & $67-89$ & $1,68-2,48$ & {$[31]$} \\
$\mathrm{In}_{2} \mathrm{O}_{3} / \mathrm{Sn}$ & $\begin{array}{l}\text { Pyrosol (R) } \\
\end{array}$ & 90 & 84 & - & {$[32]$} \\
& nos échantil- & & & & \\
$\mathrm{In}_{2} \mathrm{O}_{3}$ & lons & Evap. réact. & - & $\sim 70$ & - & \\
$\mathrm{In}_{2} \mathrm{O}_{3} / \mathrm{Sn}$ & Pulv. RF & 93 & 82 & - & {$[33]$} \\
$\mathrm{Cd}_{2} \mathrm{SnO}_{4}$ & Pulv. RF & $\sim 90$ & $86-90$ & - & {$[34]$}
\end{tabular}

( $\left.{ }^{1}\right)$ Réflexion à $10 \mu$

${ }^{2}$ ) Transmission moyenne de 0,4 à 0,6 $\mu$.

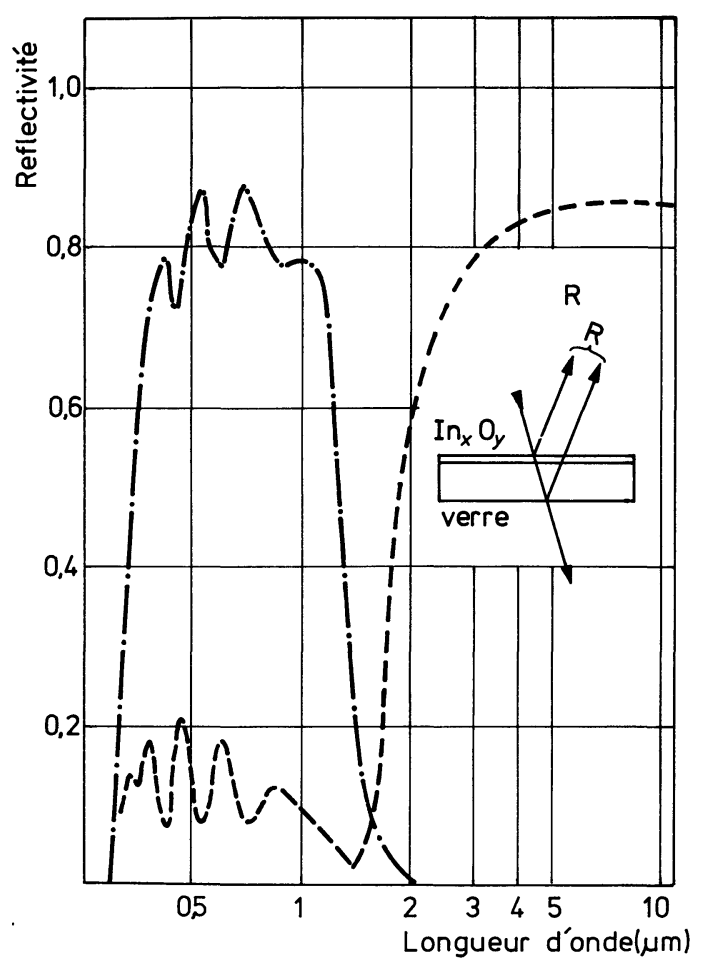

Fig. 20. - Réflectivité et transmittivité spéculaires d'une couche $\mathrm{In}_{2} \mathrm{O}_{3}$ dopé $\mathrm{S}$ déposée sur verre par procédé Pyrosol.

[Reflectivity and transmissivity of a Sn doped $\mathrm{In}_{2} \mathrm{O}_{3}$ film deposited on glass by the Pyrosol method.]
Ainsi qu'on peut le voir sur la figure 20 nos couches minces d'oxyde d'indium fortement dopé à l'étain, déposées sur verre par le procédé PYROSOL, présentent des caractéristiques optiques particulièrement intéressantes, compte tenu de l'application envisagée. Par un choix judicieux de la composition du matériau et des paramètres opératoires, nous avons pu réaliser des couches dont les coefficients de transmission solaire et de réflexion infrarouge sont respectivement égaux à 0,8 et 0,15 . De plus, cette dernière valeur est constante dans le domaine des températures inférieures à $200{ }^{\circ} \mathrm{C}$.

Tableau V. - Caractéristiques électriques et optiques de nos échantillons $\operatorname{In}_{x} \mathrm{O}_{y}$ dopé $\mathrm{Sn}$.

\begin{tabular}{|c|c|c|}
\hline Echantillon & 1 & 2 \\
\hline Epaisseur de & - & - \\
\hline $\begin{array}{l}\text { la couche } \\
(\AA)\end{array}$ & 6000 & 4200 \\
\hline $\begin{array}{l}\text { Résistivité } \\
(\Omega \mathrm{cm})\end{array}$ & $2,24 \times 10^{-4}$ & $3,87 \times 10^{-4}$ \\
\hline$e$ (ambiante) & 0,09 & 0,15 \\
\hline $\begin{array}{c}a \text { (masse } \\
\text { d'air 1) }\end{array}$ & 0,66 & 0,73 \\
\hline
\end{tabular}

7.4 Discussion. - Comme on l'a vu au paragraphe 3, il n'est pas possible de trancher en faveur de la sélectivité sur le vitrage ou sur l'absorbeur sans une connaissance précise des conditions de fonctionnement du capteur et du site où il sera implanté.

On peut donc avancer d'une manière générale que la température du vitrage étant dans tous les cas nettement inférieure à celle de l'absorbeur (Fig. 21) il apparaît judicieux d'employer des vitrages sélectifs pour les capteurs à concentration. Ainsi, les couches d'oxyde d'indium dopé ayant des caractéristiques

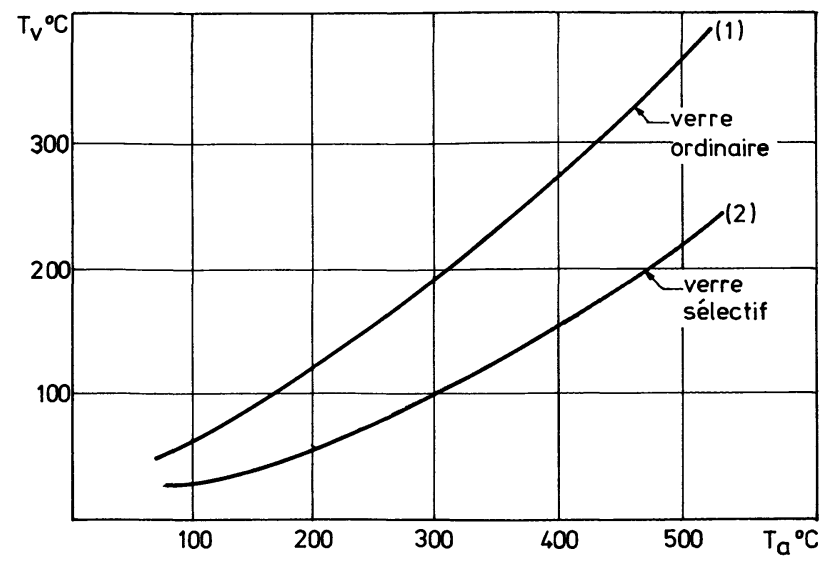

Fig. 21. - Variation de la température du vitrage en fonction de la température de l'absorbeur dans le cas des capteurs opérant sous vide.

[Glass temperature variations versus absorber temperature for collectors operating under vacuum.] 
optiques pratiquement inchangées jusqu'à $200^{\circ} \mathrm{C}$, pourront équiper sans détérioration des capteurs opérant sous vide à $500^{\circ} \mathrm{C}$.

8. Conclusion. - Le développement de la conversion photothermique de l'énergie solaire est lié, comme on vient de le voir, à l'obtention de surfaces sélectives, stables dans le temps et d'un coût acceptable.

S'il existe déjà un certain nombre de solutions techniques pour la conversion à moyenne température il apparaît clairement qu'un important effort reste à faire pour acquérir une meilleure compréhension des phénomènes conduisant à la sélectivité spectrale et réaliser des surfaces sélectives adaptées à la conversion à haute température.

Cet effort de recherche devrait s'orienter principalement dans deux directions :
- essayer de modéliser de façon plus systématique et en partant des phénomènes physiques mis en jeu, les propriétés optiques des surfaces sélectives. En effet, celles-ci ont souvent été mises au point de manière empirique ;

- étudier l'évolution des propriétés optiques des différents matériaux avec la température, en distinguant les effets dus à des changements de structure : diffusion interfaciale, réactions chimiques... ou à une contamination superficielle et les effets intrinsèques reliés aux modifications de la structure électronique.

Cet effort, faisant appel à la fois à une connaissance approfondie des propriétés optiques des solides et à des techniques de caractérisation physico-chimiques variées, devrait permettre d'améliorer les solutions existantes et d'en trouver de nouvelles, en particulier pour la sélectivité à haute température.

\section{Bibliographie}

[1] TABOR, H., Selective Radiation I. Wavelength discrimination. Bull. Res. Counc. Israel 5A (1956).

[2] Roea, Th., SPITz, J., Rapport DMG/LEMM No 3376.

[3] Mattox, D. M., J. Vac. Sci. Techn. 13 (1976) 127.

[4] Seraphin, B. O. et Meinel, A. B., Photothermal Solar Energy Conversion and the Optical properties of solids in Optical Properties of Solids. New-Developments B. O. Seraphin Ed. (North Holland Publishing Co. Amsterdam), 1976, 927.

[5] MaXwell-Garnett, Phil. Trans. R. Soc. 203 (1904) 395.

[6] Cohen, R. W., Cody, G. D., Coutts, M. D., Abeles, B., Phys. Rev. B 8 (1973) 3689.

[7] Hottel, H. C., Unger, T. A., Solar Energy 3 (1959) 10.

[8] Mattox, D. M., Sowell, R. R., J. Vac. Sci. Techn. 11 (1974) 793.

[9] Tabor, H., Harris, J., Weinberger, H., Doron, B., Proceedings of U.N. Conference on New Sources of Energy 4 (1964) 618

[10] Petersen, P. E., Ramsey, J. W., J. Vac. Sci. Techn. 12 (1975) 174.

[11] McDonald, G. E., Solar Energy 17 (1975) 119

[12] Driver, P. M., Jones, R. W., RidDiford, C. C., Simpson, R. J., Solar Energy 19 (1977) 301.

[13] Schmidt, R. N., ParK, K. C., Appl. Opt. 4 (1956) 917.

[14] Mattox, D. M., KominiaK, G. D., J. Vac. Sci. Techn. 12 (1975) 182

[15] Mahon, T. D., Jasperson, S. N., Appl. Opt. 13 (1974) 2750.

[16] Harding, G. C., J. Vac. Sci. Techn. 13 (1976) 1070.

[17] WOOD, F., BLICKENDERFER, R., Thin Solid Films 39 (1976) 133.

[18] Lincoln, R. L., Deardoff, D. K., Blickensderfer, R., S.P.I.E. 68 (1975) 161
[19] GuREv, H. S., Proceedings of the Annual Meeting of American Section of ISES, paper 5-5 (1977).

[20] Koltun, M. M., Faizev, S. A., Geliotekhnika 10 (1974) 58.

[21] Holland, J., Siddal, G., Brit. J. Appl. Phys. 9 (1958) 359.

[22] Fan, J. C. C., Bachner, F. J., Foley, G. H., Zaurack y, D. M., Appl. Phys. Lett. 25 (1974) 693.

[23] Fan, J. C. C., BaChner, F. J., Appl. Opt. 15 (1976) 1012.

[24] Horwitz, C. M., Opt. Commun. 11 (1974) 210.

[25] Fan, J. C. C., Bachner, J. J., Murphy, R. A., Appl. Phys. Lett. 28 (1976) 440.

[26] Bliaux, J., Devin, B., Besnard, C., Body, Y., Tanteri, R., WIRGIN, A., Colloque International sur l'électr. solaire, Toulouse (1976).

[27] Vossen, J. L., Transparent Conducting Films in Physics of Thin Films, ed. G. Hass, M. F. Francombe, A. W. Hoffmann (Acad. Press) 1977, vol. 9.

[28] Kane, J., Schweizer, H. P., Kern, W., J. Electrochem. Soc. 122 (1975) 1144.

[29] Kane, J., Schweitzer, H. P., Kern, W., J. Electrochem. Soc. 123 (1976) 270

[30] KANE, J., Brevet U.S. 3854.992 (1974).

[31] Kane, J., Schweizer, H. P., Kern, W., Thin Solid Films 29 (1975) 155.

[32a] SpITZ, J., VIGUIE, J. C., Brevet français no 2.110.622 (1972).

[32b] Blandenet, G., Lagarde, Y., SpItz, J., Proceedings of the Fifth International Conference on Chemical Vapor Deposition. (Eds. J. M. Blocher, H. E. Hintermann, L. H. Hall) p. 681, Electrochem. Soc. Princeton, New-Jersey (1975).

[33] ZaRZebski, Z. M., Oxide semiconductors (Pergamon Press) 1973.

[34] HaAcke, G., Appl. Phys. Lett. 30 (1977) 380. 